\title{
Quantitative experimental assessment of hot carrier enhanced solar cells at room temperature
}

\author{
Dac-Trung Nguyen ${ }^{1}$, Laurent Lombez ${ }^{1}$, François Gibelli ${ }^{1}$, \\ Soline Boyer-Richard ${ }^{2}$, Olivier Durand ${ }^{2}$ and Jean-François Guillemoles ${ }^{1}$
}

1. CNRS-Institut Photovoltaique d'Ile de France (IPVF), UMR 9006, 30 route départementale 128, 91120 Palaiseau, France.

2. FOTON-OHM, UMR 6082 CNRS-INSA, Rennes, France.

Corresponding author : laurent.lombez@cnrs.fr

\begin{abstract}
In common photovoltaic devices, the part of the incident energy above the absorption threshold quickly ends up as heat, which limits their maximum achievable efficiency far below the thermodynamic limit for solar energy conversion. Conversely, if the excess kinetic energy of the photogenerated carriers could be converted into additional free energy, it would be possible to approach the thermodynamic limit. This is the principle of hot carrier devices. Unfortunately, such a device operation in conditions relevant for utilisation has never been evidenced. Here we show that the quantitative thermodynamic study of the hot carrier population, with luminance measurements, allows us to discuss the hot carrier contribution to the solar cell performance. We demonstrate that voltage and current can be enhanced in a semiconductor heterostructure due to the presence of the hot carrier population in a single InGaAsP quantum well at room temperature. These experimental results substantiate the potential of increasing photovoltaic performances in the hot carrier regime.
\end{abstract}

\section{Introduction}

Despite their current record power conversion efficiencies (PCE) [1], solar cells are still far from their performance limits [2]. In a silicon solar cell, the mainstream technology, about $40 \%$ of the absorbed light energy is lost by thermalisation. This one of the main reasons explaining why the maximum achievable $\mathrm{PCE}$ of classical photovoltaic device is governed by Shockley-Queisser (SQ) limit of about 31\% [3]. Most of the efforts towards reaching the highest efficiencies possible are based on multijunction solar cells where each individual junction converts a fraction of the solar spectrum: in this approach the average energy converted per junction is modest while full device exhibits a substantial sensitivity to variations of the incident light spectrum. In theory, according to Ross and Nozik [4], a single diode device can be as efficient as a multijunction device if dissipation of the carrier energy via heat can be avoided (i.e. if kinetic energy of the photogenerated hot carriers could be converted into additional free energy). Indeed thermoelectric devices convert heat gradients into electrical energy and photovoltaic devices convert light flux 
into electrical energy. Hight lattice thermal conductivity of usual semiconductors would generally prevent a direct use of thermoelectric effect [6]. To fight lattice conductivity one option is to keep the lattice isothermal while only the photogenerated carriers can become hot $[4,8]$. Such decoupling of the lattice and carrier temperature has been observed in nanostructures, such as Quantum Well (QW) structures that have shown slower carrier cooling rates as compared to bulk materials [9-14].

Today we find a plethora of promising research on the development and characterisation of hot carrier absorber [15-22]. However, the optical methods used to obtain carrier temperature can be inaccurate when dealing with nanostructures and high excitation fluxes [23-25]. Meanwhile, several studies have been performed towards completed devices; especially electrical investigation to validate energy selective contacts [26-28,38].

In this work we study a hot carrier device operating at room temperature under continuous wave illumination with direct measurement of the hot carrier population and assessment of its contribution to power conversion. For this purpose we fabricated a quantum-well-based $\mathrm{p} / \mathrm{i} / \mathrm{n}$ hot carrier solar cell prototype and investigated its optical and electrical properties. To investigate hot carriers effects, we have analysed photoand electroluminescence (PL and EL) spectra from which one may extract thermodynamic quantities such as the temperature and the chemical potential of radiation (using the generalized Planck radiation law $[29,30]$ ); both are related to the carrier thermodynamic properties. We use a model of the absorption including excitons, free carriers and band filling, which allows us to fit the whole PL spectra over a large range of excitation power $[24,31]$. Quantitative optical measurements indicate the presence of a hot carrier population within the quantum well. This gives an increase of the electrochemical potential in the barrier. Such an effect is analogous to a Seebeck conversion process [20]. The quantitative optical measurements are then compared to the electrical characteristics. The real impact of the hot carrier population within the structure is then discussed and quantified.

\section{Quantum well hot carrier solar cell}

The solar cell prototype is depicted in Figure 1. The multilayer wafer contains a region with an intrinsic InGaAsP-based quantum well/barriers, which acts as the absorber generating hot carriers, while the barriers and claddings play the role of energy semiselective contacts $[16,32]$ (see Table 1). This absorber-contacts layer is sandwiched between two gradually $n$ - and $p$-doped InP layers for carrier separation and collection.

Current-voltage characteristics are first recorded for cells with diameters ranging from 5 to $200 \mu \mathrm{m}$. The results for the $11.0 \pm 0.5 \mu \mathrm{m}$ diameter cell shows the highest efficiency under $\mathrm{cw}$ laser excitation $980 \mathrm{~nm}$. The PCE is $11.6 \pm 0.5 \%$ under focused laser illumination of $1650 \mathrm{~W} / \mathrm{cm}^{2}$ (equivalent to 55600 Suns and close to the maximum achievable solar concentration, see Supplementary Notes 4 to 6 for more details). Although we measure efficiencies of potential hot carrier solar cell (HCSC), the purpose of the design is to allow a proper optoelectrical measurements. The excellent electrical characteristics stem from an extremely low series resistance, which yields a short-circuit 

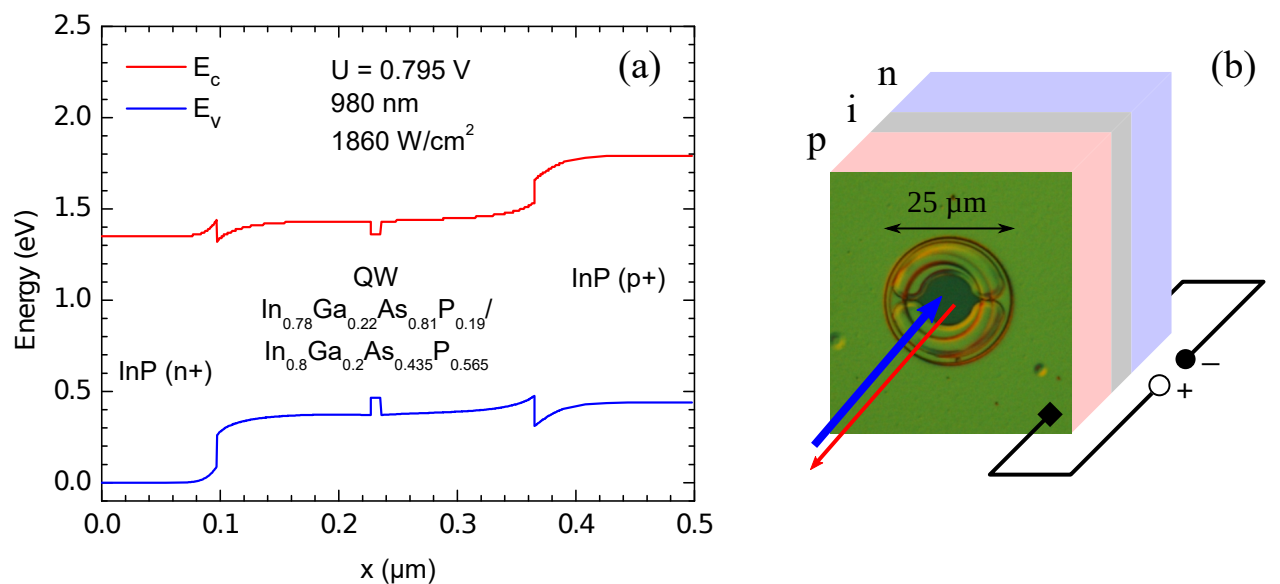

Figure 1: Description of the hot carrier heterojunction device. (a)Energy band diagram of the heterostructure with $980 \mathrm{~nm}$ excitation wavelength, $1890 \mathrm{~W} / \mathrm{cm}^{2}$ and at open circuit condition. $\mathrm{E}_{c}\left(\mathrm{E}_{v}\right)$ stands for conduction (valence) energy band level. (b) Top view of the micro-solar cell taken with an optical microscope(golden green) and schematic of the opto-electrical characterisation (light red - grey - light blue). Charge carriers are generated by $\mathrm{cw}$ laser excitation at $980 \mathrm{~nm}$ (blue arrow) and thermodynamic properties of charge carriers are probed by the luminescence induced by radiative recombination (red arrow).

\begin{tabular}{cccc} 
Composition & Doping & Thickness & Role \\
\hline $\operatorname{InGaAs}$ & $\mathrm{p}++$ & $170 \mathrm{~nm}$ & Electric contact \\
$\mathrm{In}_{0.8} \mathrm{Ga}_{0.2} \mathrm{As}_{0.435} \mathrm{P}_{0.565}$ & $\mathrm{p}+$ & $50 \mathrm{~nm}$ & \\
$\operatorname{InP}$ & $\mathrm{p}+$ & $50 \mathrm{~nm}$ & Cladding \\
$\mathrm{In}_{0.8} \mathrm{Ga}_{0.2} \mathrm{As}_{0.435} \mathrm{P}_{0.565}$ & $\mathrm{i}$ & $120 \mathrm{~nm}$ & Barrier \\
$\mathrm{In}_{0.78} \mathrm{Ga}_{0.222} \mathrm{As}_{0.81} \mathrm{P}_{0.19}$ & $\mathrm{i}$ & $7.4 \mathrm{~nm}$ & Quantum Well \\
$\mathrm{In}_{0.8} \mathrm{Ga}_{0.2} \mathrm{As}_{0.435} \mathrm{P}_{0.565}$ & $\mathrm{i}$ & $130 \mathrm{~nm}$ & Barrier \\
$\operatorname{InP}$ & $\mathrm{n}+$ & $401 \mu \mathrm{m}$ & Cladding
\end{tabular}

Table 1: Table detailing the composition layers. The intrinsic (i) InGaAsP-based absorber consists of a $7.4 \mathrm{~nm}$ quantum well $\left(E_{\mathrm{g}}=0.78 \mathrm{eV}\right)$ between 120 and $130 \mathrm{~nm}$ barriers $\left(E_{\mathrm{g}}=1.05 \mathrm{eV}\right)$. The absorber is sandwiched between p- and n-doped InP contact layers. 
current that is proportional to the laser power within the entire experimental range (see Figure 2). The open circuit voltage exceeds the lowest absorption threshold at laser fluences higher than $\sim 6000 \mathrm{~W} / \mathrm{cm}^{2}$, which would indicate a power conversion efficiency above an equivalent thermal equilibrium device. In order to determine whether band filling contributes solely to this high conversion efficiency, further analysis are required as laid out in the following.
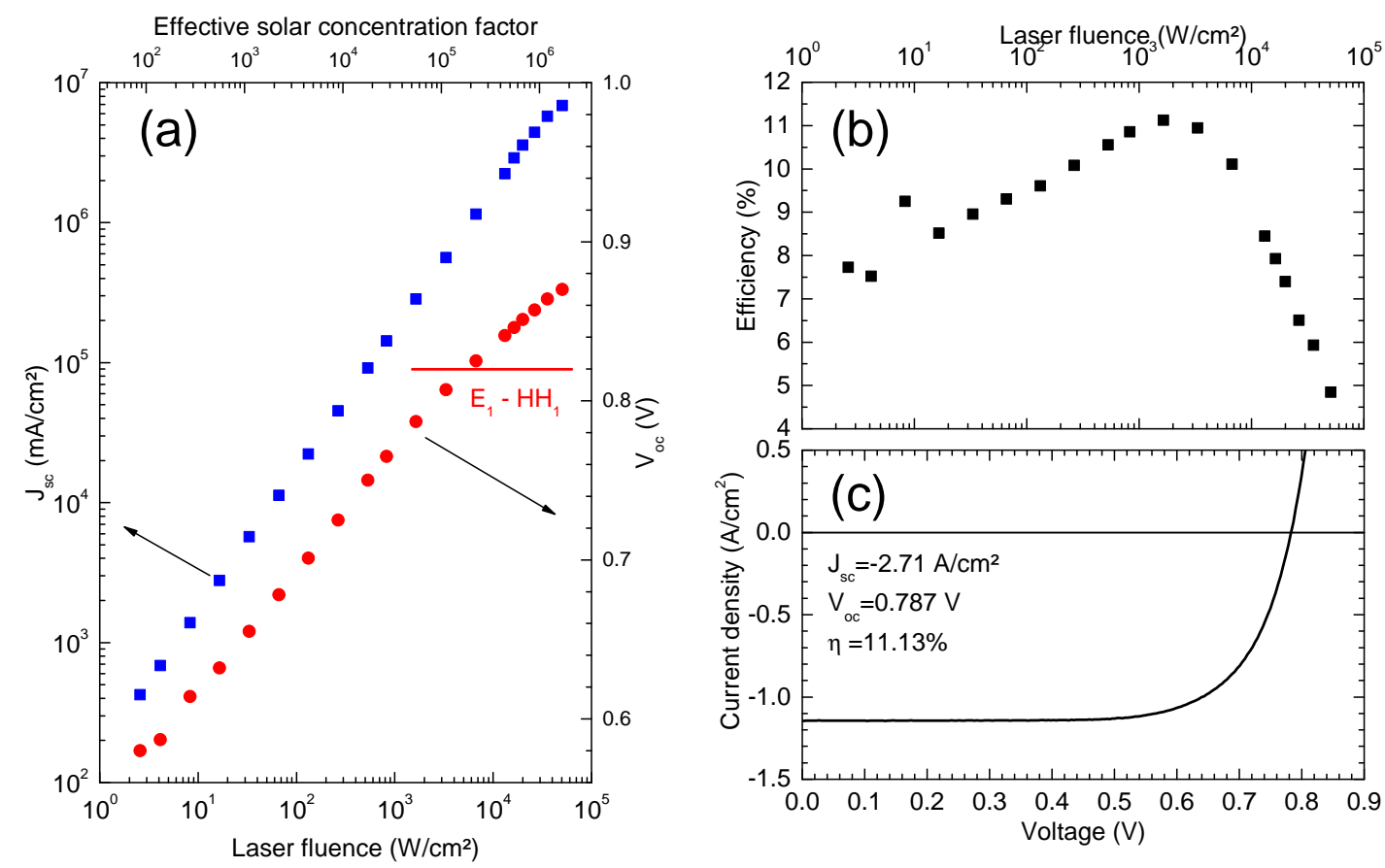

Figure 2: Electrical characteristics of the heterojunction device. Variation of short-circuit current density ( $J_{\mathrm{sc}}$, blue squares) and open-circuit voltage ( $V_{\mathrm{oc}}$, red circles) with laser power. The red horizontal line indicates the $E_{1}-H H_{1}$ transition energy. The indicative solar illumination concentration incident on the device is obtained by multiplying the laser fluence (in $\mathrm{W} / \mathrm{cm}^{2}$ ) by a factor of 33.7 , as $J_{\mathrm{sc}}=4.5 \mathrm{~mA} / \mathrm{cm}^{2}$ was measured under AM1.5 solar spectrum excitation. Details on this conversion are given in the Supplementary Note 6. (b) Equivalent conversion efficiency as a function of the effective concentration with $980 \mathrm{~nm}$. (c) current-voltage curve taken at the maximum efficiency.

\section{Determination of carriers thermodynamic properties}

Luminescence spectra, calibrated in absolute units, are recorded at room temperature and atmospheric pressure in order to determine the carrier thermodyamic properties. 
Indeed, the carrier temperature $T$ as well as the quasi-Fermi level splitting $\mu=E_{\mathrm{Fn}}-E_{\mathrm{Fp}}$ ( $E_{\mathrm{Fn}}$ and $E_{\mathrm{Fp}}$ being the electrons and holes Fermi levels) are determined according to the generalized Planck's law $[5,24,30]$ :

$$
\Phi(E)=\frac{2 A(E) \cdot E^{2}}{h^{3} c^{2}} \cdot \frac{1}{\exp \left(\frac{E-\mu}{k_{\mathrm{B}} T}\right)-1}
$$

Here $\Phi$ is the luminescence emission, $E$ the photon energy, $A(E)$ the absorptivity, $h$ the Planck's constant, $c$ the speed of light in vacuum and $k_{\mathrm{B}}$ the Boltzmann constant.

Determination of the absorptivity $A(E)$ is crucial for minimizing the uncertainty in the evaluation of the thermodynamic properties $T$ and $\mu$ in the equation 1 (see section Methods and the Supplementary Notes 1 and 2). Indeed $A(E)$ might affect the whole spectral variation of the PL signal including the high energy range. In order to validate the model of the absorption in the sample structure, we compare the absorptivity calculated from luminescence spectra with the external quantum efficiency EQE (see Figure 3). Indeed, if the collection of photo-generated carriers was lossless, the electric current density would correspond to the number of absorbed photons [29]. In the case of relatively shallow wells, as in our samples $\left(\Delta E_{\mathrm{c}} \lesssim 0.2 \mathrm{eV}\right)$, the collection efficiency is generally found close to unity [33-35]. Hence photocurrent can be used as an indirect measure of the absorption. We found a good agreement between the EQE and $A(E)$ obtained from our model.

Therefore, luminescence spectra at different laser excitation powers and different applied voltages have been recorded and analysed. In all cases the full-spectrum fit is in good agreement with the experimental data as can be seen in Figures 4 and 5 .

We optically probe the carrier thermodynamic properties in both the barrier and the QW spectral regions (see Supplementary Note 7 for detailed results). Figure 4c shows the carrier temperature as a function of the excitation power. We observe a temperature increase of more than $1300 \mathrm{~K}$ above the room temperature for carrier in the QW (i.e $1300 \mathrm{~K}$ above the carrier temperature in the barrier) - a range of temperature already reported in the litterature $[23,25]$. We have previously demonstrated that this surplus amount of heat can be converted into a gain in voltage via a Richardson emission of hot carriers (also seen as a pseudo-Seebeck effect) [20]. This is confirmed when looking at the electrochemical potentials of the carriers in both spectral region (see Figure 4d): The difference between the two values increases with the carrier temperature in the quantum well. Notably, the value of the electrochemical potential $\mu_{\mathrm{b}}$ in the barrier region exceeds the lowest absorption threshold of the $\mathrm{QW}(0.82 \mathrm{eV})$, which is consistent with the electrical response we observe.

The determined absorptivities for three laser excitation powers are shown in Figure $4 \mathrm{~b}$. One can infer that saturation of absorption in the quantum well region is only partial, which indicates that the quantum well would be still optically active for absorption. This observation goes with the hypothesis that the increase of the electrochemical potential in the quantum well $\mu_{\mathrm{b}}$ is linked to a hot carrier effect rather than a band-filling phenomenon. Therefore the fact that $\mu_{\mathrm{b}}$ is higher than the fundamental transition $\mathrm{E}_{1^{-}}$ 


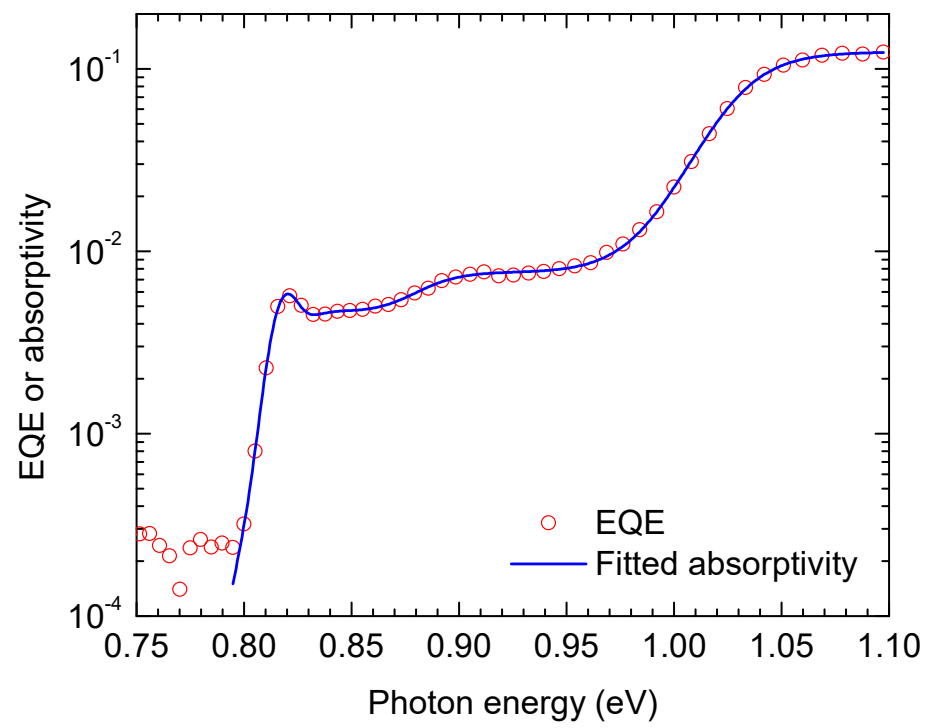

Figure 3: External quantum efficiency (EQE) and absorption model. Data are shown from $0.78 \mathrm{eV}(1550 \mathrm{~nm})$ to $1.10 \mathrm{eV}(1127 \mathrm{~nm})$ where absorption by the quantum well/barriers occurs. The EQE (red circles) is fitted using the expression of unsaturated absorptivity (blue line) $A_{0}(E)=(1-R)\left\{1-\exp \left[-\left(\alpha_{\mathrm{w} 0} d_{\mathrm{w}}+\alpha_{\mathrm{b} 0} d_{\mathrm{b}}\right)\right]\right\}$. The reflectivity $R$ is measured to be about 0.3 over the spectral range $[0.75,1.10] \mathrm{eV}$. The absorption model includes quantum well interband excitonic and free carrier absorption (in $\alpha_{\mathrm{w}}$ ), as well as barrier interband absorption (in $\alpha_{\mathrm{b}}$ ). See Supplementary Note 1 for more details on the quantum/well absorption coefficients $\alpha_{\mathrm{w} 0}$ and $\alpha_{\mathrm{b} 0}$. The experimental data reflects both qualitatively and quantitatively the absorptivity obtained from PL measurements at low excitation power. The exciton peak is centred at $0.82 \mathrm{eV}$ and the absorption plateaus are observed, corresponding to the fundamental and the first excited levels of the quantum well. The detailed result is presented in the Supplementary Note 3. 

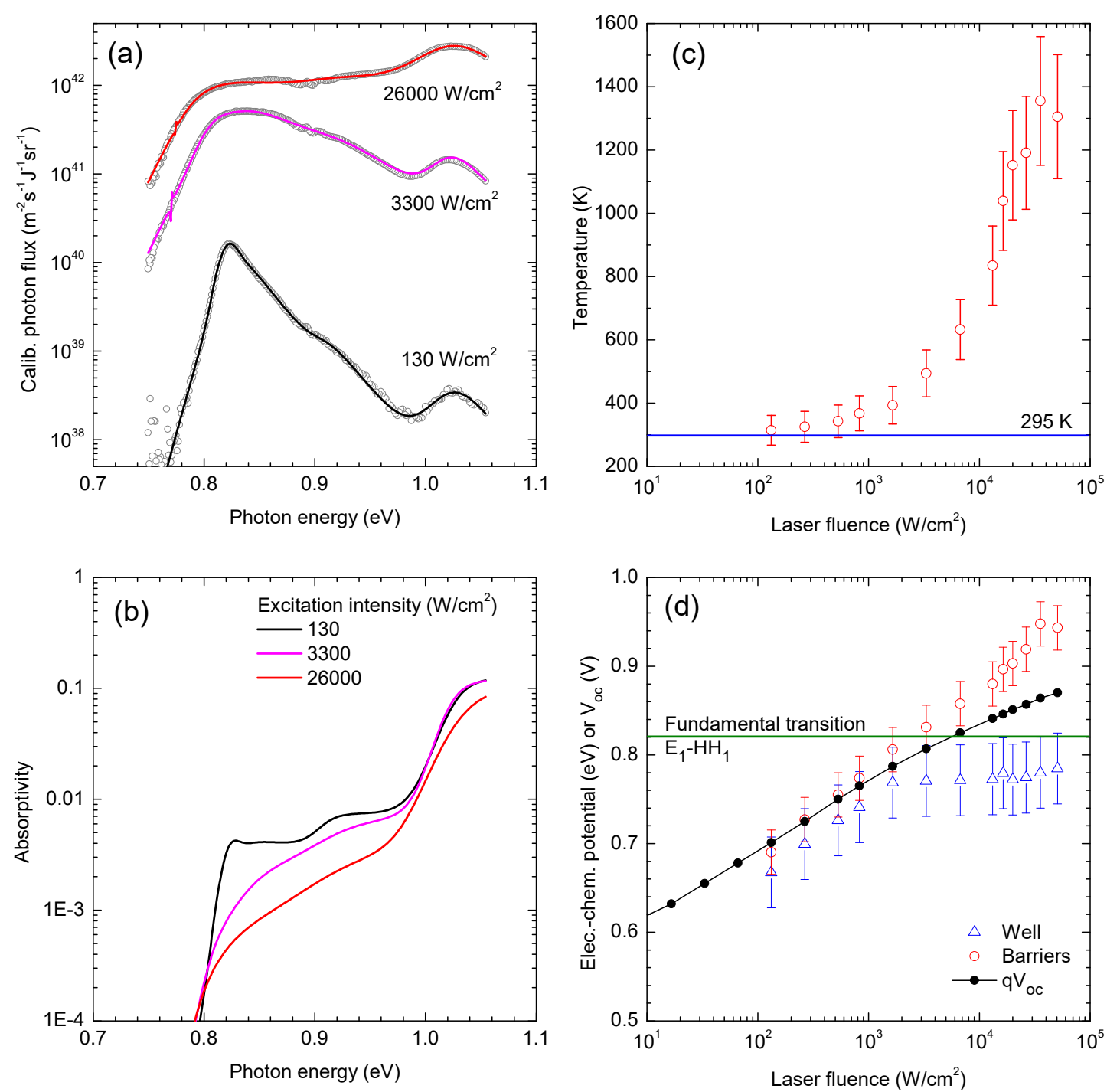

Figure 4: Variation of carriers thermodynamic properties with laser intensity. (a) Three PL spectra (grey circles) and fit at low (black line), medium (magenta line) and strong (red line) laser excitation, in open circuit regime. (b) : Corresponding absorptivity deduced from fit. (c) and (d): Variation of quantum well carrier temperature $T_{w}$ and electrochemical potentials in quantum well $\mu_{\mathrm{w}}$ (red circles) and barriers $\mu_{\mathrm{b}}$ (blue triangles) with laser power. Evaluated uncertainties in the optical measurement are displayed by errors bars. See Methods section for details on the evaluation of uncertainties. 
$\mathrm{HH}_{1}$ would indeed indicate the barriers are allowing for isentropic cooling of the carriers which corroborates hot carrier solar cell functionality.

We then carried out luminescence experiments under electric bias to quantify the contribution of this hot carrier effect in the obtained device characteristics. Similarly to Figure 4 we display in Figure 5 fits of biased-PL spectra, absorptivity and evolution of the thermodynamic quantities with the applied voltage (see Supplementary Note 8 for detailed results). The incident laser excitation power is set to $1890 \mathrm{~W} / \mathrm{cm}^{2}$. All the measurements are carried out in a collection regime, at applied bias lower than the $V_{\mathrm{oc}}=0.795 \mathrm{~V}$. In such optoelectrical experiment, one might expect values of $\mu_{\mathrm{b}}$ close to the applied bias as the carriers in this spectral region are supposed to be cold (i.e. fast thermalisation). The green line in Figure 5 d shows the condition where $V=\mu / q$. The electrochemical potential $\mu_{b}$ in our case is found to be slightly higher than the applied voltage (see Figure 5d blue triangles) and two different regimes can be distinguished. Below a bias voltage $V=0.6 \mathrm{~V}$ we see a rather constant value of $m u_{b}$ around 0.7 $\mathrm{eV}$ which then increase for higher bias voltages. This can be explained by a weak carrier collection efficiency at high excitation intensities. The effect is confirmed by the observation of a PL signal at all biases, although all the carriers would have been electrically collected (see Figure 5d blue lines). The luminescence signal under bias is the sum of the electroluminescence and PL signal at short circuit current [29], the latter being small but non negligible in our case.

Fixing the laser intensity to a high value and starting at the open-circuit condition, we would, expect $T$ and $\mu$ to decrease in the quantum well region with decreasing bias voltage (i.e. we reduce the carrier density by extracting carriers as expected) as illustrated by the results presented in Figure 5 as the bias voltage is varied from $0.8 \mathrm{~V}$ to $0.6 \mathrm{~V}$. Thermodynamic values below $0.6 \mathrm{~V}$ are once again affected by a reduced carrier collection efficiency.

\section{Contribution of hot carriers to voltage and current gains}

A key point in the latter experiment is to probe the hot carrier effect at the maximum power point which is found at $0.651 \mathrm{~V}$ (grey zone highlighted in Figure 5d). With the laser excitation condition used here $\left(1890 \mathrm{~W} / \mathrm{cm}^{2}\right.$ on a $11 \mu \mathrm{m}$-diameter cell) we obtain a PCE of $11.6 \%$. Here, the electrochemical potential increase from $\mu_{\mathrm{w}}$ to $\mu_{\mathrm{b}}$ by 110 $\mathrm{mV}$, and $50 \mathrm{mV}$ for the maximum power point showing an efficient conversion of the temperature difference into electrochemical potential difference [20]. Still, we need to estimate the hot carrier contribution to the total voltage and current.

In a first step approach we propose to compare the open circuit voltage in a hot and cold carrier regime. We assess the contribution of the well to the power conversion as a function of hot carriers' temperature, using the fact that the hot carriers are essentially in the well. Two situations are considered: (i) the experimental scenario for which hot carriers are produced in the well, and where electrochemical potential is known in the barriers, and (ii) a virtual situation in which the carriers in the well would be cold and in equilibrium with the barrier. This situation can be simulated using our fitting 

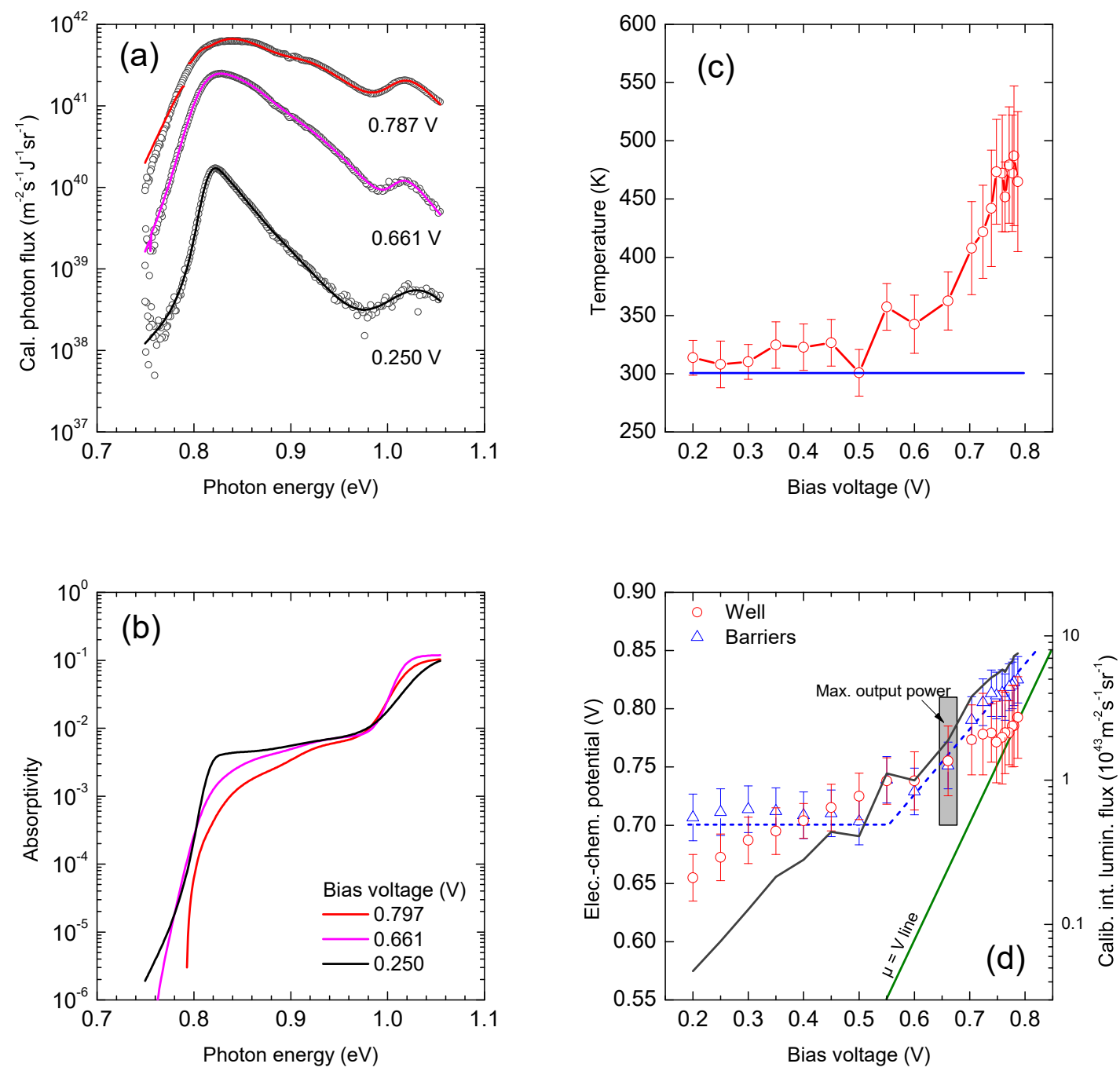

Figure 5: Variation of carriers thermodynamic properties with bias voltage. The laser intensity is at $1890 \mathrm{~W} / \mathrm{cm}^{2}$. (a): Three PL spectra (grey circles) with respective fit (continuous lines) at three bias voltages $-V=0.250 \mathrm{~V}$ (black line), $V=0.661 \mathrm{~V}$ (magenta line) and $V=0.787 \mathrm{~V}$ (red line). (b): Corresponding absorptivity deduced from fit at the same bias voltages. (c): Variation of carrier temperature in quantum well (red circles) with bias voltage. The blue line represents ambient temperature around 297 K. (d): Variation of electrochemical potential in quantum well (red circles) and barriers (blue triangles), and of luminescence intensity (black continuous line) with bias voltage. The dashed blue line serves as a visual guide for the variation of the barriers' electrochemical potential and the green continuous line depicts the $\mu=V$ dependence. The grey box highlights the maximum output power working point. The evaluation of uncertainties is detailed in Methods section. 
parameters (see Supplementary Note 9). Figure 6(a) shows the evolution of $\mu_{b}$ (i.e. maximum achievable voltage for the case of hot carriers) and the calculated one $\mu_{\text {cold }}$ for the case of cold carriers. We assumed here the same radiative efficiency in both cases and find that the quasi Fermi level splitting in the barriers to be larger by about 25 to 30 meV with hot carriers as compared to the case of lattice thermalized carriers (see Figure $6(a)$ ). The same simulation in the experiment under bias (and lower laser excitation power ) shows an increase of the QFLS by about $10 \mathrm{meV}$ for $\mathrm{V}>0.65 \mathrm{~V}$ (i.e. where hot carriers are present). This is a strong indication of a voltage gain from the conversion of the hot carriers' thermal energy.

In the next step we probe whether there is any possible gain in current. Although the experiments presented here give a clear picture of the physical phenomena at work, they do not exactly correspond to the operation of a solar cell. To understand the impact of hot carriers in a heterostructure on the conversion of solar energy, it is necessary to extrapolate the previous describes case i.e. the laser only excites transitions in the barriers, to the case where the solar spectrum excites all optical transitions. In the former case, transitions from the well only contribute to the recombination current while in the latter case they also contribute to the photocurrent. The key point is whether the balance between the additional generation and recombination due to the presence the well yields a positive contribution to solar energy conversion or not.

In order to assess the contribution of the well to current generation, we propose as a metric to consider the marginal gain (additional generation) versus the marginal loss (additional recombination) induced by the well at a given operating point. Subsequently, we evaluate this metric in the presence as well in the absence of hot carriers. All the procedure is detailed in the Supplementary Note 9. On the one hand, the marginal generation is computed for a solar spectrum at a concentration yielding the same photocurrent as measured at the considered working point. We use the measured absorptivity in the spectral range where only well transitions contribute (e.g. $\mathrm{E}<1.1 \mathrm{eV}$ ). As shown in Figure 6 (b) the photogenenated generation current increases in the presence of hot carriers and is always higher than the generation current in the equilibrium case (i.e. cold carriers). In that case, the current vanishes because of the net absorption reduction where stimulated emission could occur (i.e. strong band filling effect) [5].

On the other hand, the marginal recombination was computed from radiative recombination assuming a radiative yield (see Figure 6(c)). The recombination current increases in both the hot and the cold regime with the cold regime yielding higher values. Finally to probe what would be the contribution of current due to the quantum well in case of a solar spectrum illumination, we need to subtract the generation current from the recombination current. This gain is much more pronounced in the hot regime. Therefore, the presence of hot carriers is essential for the QW region to act as a current generator.

This finding is very promising since the present structure suffers from weak absorption in the barrier and more importantly, in the single well (which collects only $\lesssim 1 \%$ of the incident light, see Figures $4 \mathrm{~b}$ and $5 \mathrm{~b}$, a value comparable to monolayers of $2 \mathrm{D}$ materials such as graphene). Here, light management in the cell would be beneficial for reducing 

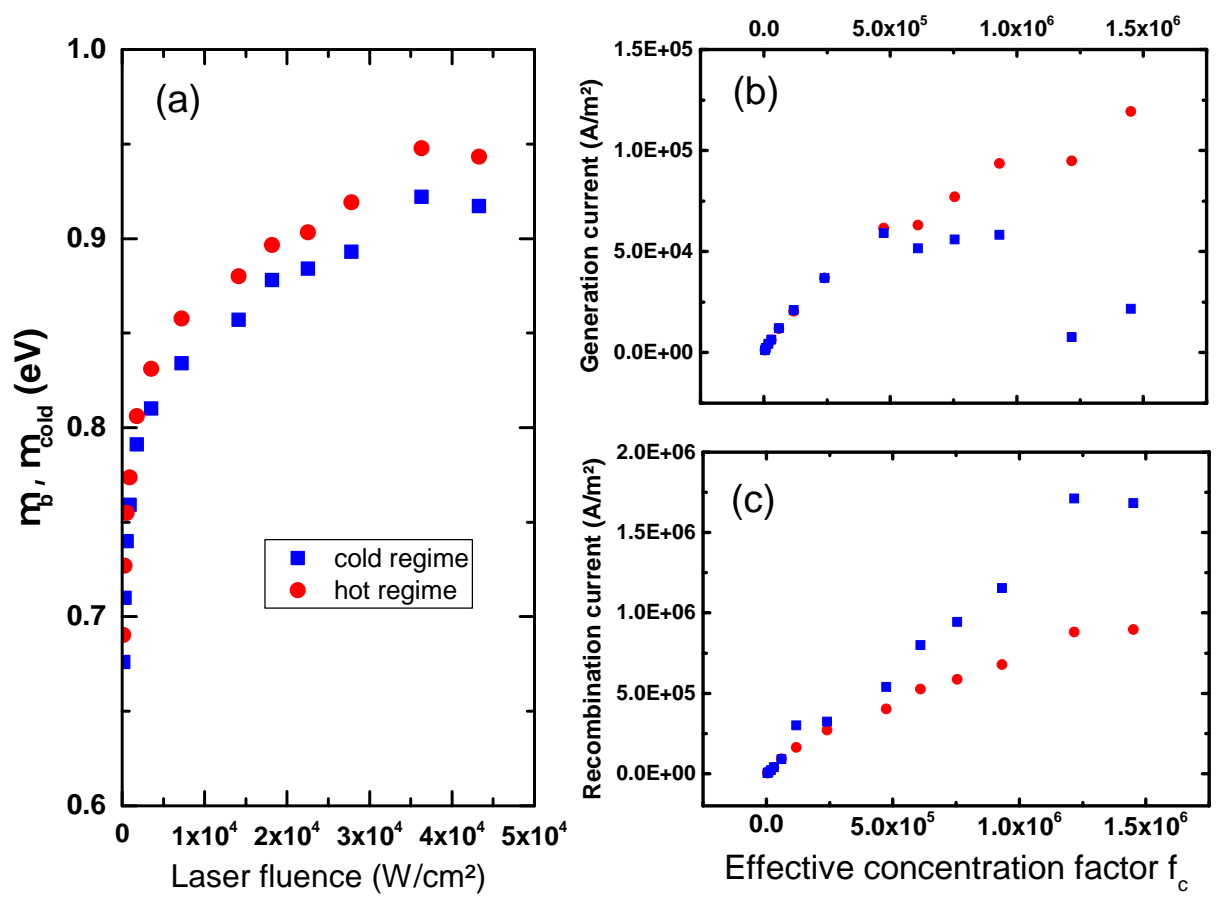

Figure 6: Assessment of the hot carriers' contribution to power generation . Evaluation of the hot carrier contribution on the electrical properties of the cell with the excitation intensity. (a) Evolution of the electrochemical potential (b) generation current and (c) recombination current in the hot carrier regime (red dots) and cold carrier regime (blue square).

the effective concentration for the same amount of generated current and thus increase the impact of the QW to solar energy conversion.

\section{Conclusion}

We investigated $\mathrm{p} / \mathrm{i} / \mathrm{n}$ single quantum well device by means of complementary optical and electrical measurements. Our device exhibit its maximum efficiency at incident powers equivalent to 50000 suns, i.e. one order of magnitude higher than current achievement in concentrator photovoltaics. Electrical measurements show Voc above the minimum absorption threshold that could indicate a working condition of this device above the limit for classical device operation where thermal equilibrium is present. To clarify this observation, the variation of the carrier thermodynamic properties as a function of optical excitation power or electrical bias is determined by rigorous fit of 
the complete luminescence spectra. The optical measurements confirm the electrical behavior and allow to quantify the impact of hot carriers on device functionality. A striking observation is that voltage and current enhancement are observed due to the presence of hot carriers. These findings exemplify that current state-of-the-art semiconductor heterostructures can already be used to boost solar energy conversion by hot carrier mechanisms. The present results will be instrumental to designing a new generation of devices for enhancing the hot carrier conversion contribution, both optically and electrically.

\section{Methods}

\section{Sample preparation and hyperspectral imaging}

The investigated sample is based on a quaternary $\operatorname{In}_{x} \mathrm{Ga}_{1-x} \mathrm{As}_{y} \mathrm{P}_{1-y}$ compound grown on lattice-matched InP substrate. Layer composition and thicknesses are given in Figure 1. The wafer structure has been optimized from the results of our previous work [6] which optically showed the evidence of hot carrier effects in a quantum well/barriers absorber. The multilayer wafer contains an intrinsic InGaAsP-based quantum well/barriers region, playing the role of absorber which generates hot charge carriers. The barriers play the role of semi-selective contacts where the carriers are mainly cold. This well/barriers region is sandwiched between two gradually $n$ - and $p$-doped InP layers for carriers separation and collection. The quantum well has been designed to minimize the number of energy levels in the QW and to spectrally separate absorption in the quantum well and in the barriers, to simplify the spectral analysis. Band structures calculating from k.p formalism is shown in Supplementary Figure 1 indicating that a single optical transition is dominant in the QW region.

In our experimental setup, a hyperspectral imaging system from Photon Etc. records quantitative luminescence spectra (luminance), which we subsequently calibrate to obtain absolute values. This allows us to evaluate the thermodynamic properties of the emission that is related to the carrier thermodynamic properties. The excitation laser wavelength is $980 \mathrm{~nm}$. All measurements are carried out at room temperature. More details on the experimental setup are seen in Supplementary Figure 2.

\section{Modelling the absorption}

The absorptivity is calculated from absorption coefficients and layer thicknesses. For our well/barriers structure we write:

$$
A(E)=[1-R(E)] \cdot\left\{1-\exp \left[-\left(\alpha_{\mathrm{w}} d_{\mathrm{w}}+\alpha_{\mathrm{b}} d_{\mathrm{b}}\right)\right]\right\}
$$

Where $\alpha_{\mathrm{w}}$ and $\alpha_{\mathrm{b}}$ are effective absorption coefficients of well and barriers, $d_{w}$ and $d_{\mathrm{b}}$ their thicknesses. We used an absorption model taking into account excitons and free carriers in the well as well as free carriers in the barriers $[17,19,20]$. This effect of 
band filling [37] is expressed by introducing occupation functions into the absorption coefficient $\alpha_{s}=\alpha_{0} \cdot\left[f_{\mathrm{v}}^{\mathrm{e}}\left(E_{\mathrm{h}}\right)-f_{\mathrm{c}}^{\mathrm{e}}\left(E_{\mathrm{e}}\right)\right]$. Detailed expressions of absorption are provided in the see Supplementary Notes 1 and 2.

\section{Evaluation of uncertainties in optical measurements}

Temperature $T$ and electrochemical potential $\mu$ are determined from fit of luminescence spectra. Uncertainties, in the measurement of $T$ for example, are evaluated by manually assigning it to a fixed value $T$ around its fitted value $T_{0}$ and launch the fit again, until the fitted spectrum becomes unsatisfactory. Mathematically, that means $\sim 10 \%$ or more of the points in the fitted luminescence spectrum present a relative error $\mid \Phi_{\text {fit }}(T)-$ $\Phi_{\exp } \mid / \Phi_{\exp }$ of at least $\sim 10 \%$ compared to experimental data.

\section{Data availability}

The data that support the plots within this paper and other findings of this study are available from the corresponding author upon reasonable request.

\section{Acknowledgements}

This work was carried out in the framework of a project of IPVF (Institut Photovoltaïque d'Île-de-France). This project has been supported by the French Government in the framework of the program of investment for the future (Programme d'Investissement d'Avenir) ANR-IEED-002-0. The authors acknowledge J. Even for the energy band structure simulation, and P. Schultz and J. Connolly for a careful reading of the manuscript.

\section{Authors contribution}

JFG and LL did the planning of the study; DTN did the data acquisition; DTN, LL, FG contributed to data treatment; SBR and OD did the sample fabrication; DTN, LL and JFG, contributed to data analysis, modeling and wrote the paper.

\section{References}

[1] Green, M. A. et al. Solar cell efficiency tables (version 50). Progress in Photovoltaics: Research and Applications 25, 668-676 (2017).

[2] Green, Martin A. Third generation photovoltaics (Springer-Verlag Berlin Heidelberg, 2006).

[3] Shockley, W. \& Queisser, H. J. Detailed Balance Limit of Efficiency of p-n Junction Solar Cells. Journal of Applied Physics 32, 510-519 (1961). 
[4] Ross, R. T. \& Nozik, A. J. Efficiency of hot-carrier solar energy converters. Journal of Applied Physics 53, 3813-3818 (1982).

[5] Würfel, P. The chemical potential of radiation . J. Phys. C:Solid State Phys. 15, 3967-3985 (1982).

[6] Kettemann, S. \& Guillemoles, J.-F. Thermoelectric field effects in low-dimensional structure solar cells. Physica E: Low-dimensional Systems and Nanostructures 14, 101-106 (2002).

[7] Schwede, J. W. et al. Photon-enhanced thermionic emission for solar concentrator systems. Nature Materials 9, 762-767 (2010).

[8] Würfel, P. Solar energy conversion with hot electrons from impact ionisation. Solar Energy Materials and Solar Cells 46, 43-52 (1997).

[9] Rosenwaks, Y.et al. Hot-carrier cooling in GaAs: Quantum wells versus bulk. Physical Review B 48, 14675-14678 (1993).

[10] Xu, Z. Y. \& Tang, C. L. Picosecond relaxation of hot carriers in highly photoexcited bulk GaAs and GaAs-AlGaAs multiple quantum wells. Applied Physics Letters 44, 692-694 (1984).

[11] Ryan, J. F. Time-Resolved Photoluminescence of Two-Dimensional Hot Carriers in GaAs-AlGaAs Heterostructures. Physical Review Letters 53, 1841-1844 (1984).

[12] Shah, J. Energy-Loss Rates for Hot Electrons and Holes in GaAs Quantum Wells. Physical Review Letters 54, 2045-2048 (1985).

[13] Leo, K. Reduced dimensionality of hot-carrier relaxation in GaAs quantum wells. Physical Review B 37, 7121-7124 (1988).

[14] Lugli, P. Nonequilibrium longitudinal-optical phonon effects in GaAs-AlGaAs quantum wells. Physical Review Letters 59, 716-719 (1987).

[15] Conibeer, G. et al. Modelling of hot carrier solar cell absorbers. Solar Energy Materials and Solar Cells 94, 1516-1521 (2010).

[16] Le Bris, A. \& Guillemoles, J.-F. Hot carrier solar cells: Achievable efficiency accounting for heat losses in the absorber and through contacts. Applied Physics Letters 97, 113506 (2010).

[17] Le Bris, A. et al. Thermalisation rate study of GaSb-based heterostructures by continuous wave photoluminescence and their potential as hot carrier solar cell absorbers. Energy \& Environmental Science 5, 6225-6232 (2012).

[18] Le Bris, A. et al. Hot Carrier Solar Cells: Controlling Thermalization in Ultrathin Devices. IEEE Journal of Photovoltaics 2, 506-511 (2012). 
[19] Hirst, L. C., Walters, R. J., Führer, M. F. \& Ekins-Daukes, N. J. Experimental demonstration of hot-carrier photo-current in an InGaAs quantum well solar cell. Applied Physics Letters 104, 231115 (2014).

[20] Rodière, J., Lombez, L., Le Corre, A., Durand, O. \& Guillemoles, J.-F. Experimental evidence of hot carriers solar cell operation in multi-quantum wells heterostructures. Applied Physics Letters 106, 183901 (2015).

[21] Esmaielpour, H. et al. Suppression of phonon-mediated hot carrier relaxation in type-II InAs/AlAsxSb1 - x quantum wells: a practical route to hot carrier solar cells. Progress in Photovoltaics: Research and Applications 24, 591-599 (2016).

[22] Zhang, Y. et al. Extended hot carrier lifetimes observed in bulk In0.265 \pm 0.02 ga0.735n under high-density photoexcitation. Applied Physics Letters 108, 131904 (2016).

[23] Tedeschi, D. et al. Long-Lived Hot Carriers in III-V Nanowires. Nano Letters 16, 3085-3093 (2016).

[24] Gibelli, F., Lombez, L. \& Guillemoles, J.-F. Accurate radiation temperature and chemical potential from quantitative photoluminescence analysis of hot carrier populations. Journal of Physics: Condensed Matter 29, 06LT02 (2017).

[25] Esmaielpour, H. et al. Effect of occupation of the excited states and phonon broadening on the determination of the hot carrier temperature from continuous wave photoluminescence in InGaAsP quantum well absorbers. Progress in Photovoltaics: Research and Applications 25,782-790 (2017).

[26] Dimmock, J. A. R., Day, S., Kauer, M., Smith, K. \& Heffernan, J. Demonstration of a hot-carrier photovoltaic cell. Progress in Photovoltaics: Research and Applications 22, 151-160 (2014).

[27] Dimmock, J. A. R. et al. Optoelectronic characterization of carrier extraction in a hot carrier photovoltaic cell structure. Journal of Optics 18, 074003 (2016).

[28] Julian, A., Jehl, Z., Miyashita, N., Okada, Y. \& Guillemoles, J. F. Insights on energy selective contacts for thermal energy harvesting using double resonant tunneling contacts and numerical modeling. Superlattices and Microstructures 100, 749-756 (2016).

[29] Rau, U. Superposition and Reciprocity in the Electroluminescence and Photoluminescence of Solar Cells. IEEE Journal of Photovoltaics 2, 169-172 (2012).

[30] Lasher, G. \& Stern, F. Spontaneous and Stimulated Recombination Radiation in Semiconductors. Physical Review 133, A553-A563 (1964).

[31] Gibelli, F., Lombez, L. \& Guillemoles, J.-F. Two carrier temperatures nonequilibrium generalized Planck law for semiconductors. Physica B: Condensed Matter 498, 7-14 (2016). 
[32] Feng, Y. et al. Non-ideal energy selective contacts and their effect on the performance of a hot carrier solar cell with an indium nitride absorber. Applied Physics Letters 100, 053502 (2012).

[33] Tsui, E., Nelson, J., Barnham, K., Button, C. \& Roberts, J. S. Determination of the quasi-Fermi-level separation in single-quantum-well p-i-n diodes. Journal of Applied Physics 80, 4599-4603 (1996).

[34] Kluftinger, B., Barnham, K., Nelson, J., Foxon, T. \& Cheng, T. Temperaturedependent study of the radiative losses in double-quantum well solar cells. Solar Energy Materials and Solar Cells 66, 501-509 (2001).

[35] Nelson, J., Paxman, M., Barnham, K. W. J., Roberts, J. S. \& Button, C. Steadystate carrier escape from single quantum wells. IEEE Journal of Quantum Electronics 29, 1460-1468 (1993).

[36] Araújo, G. L. \& Martí, A. Absolute limiting efficiencies for photovoltaic energy conversion. Solar Energy Materials and Solar Cells 33, 213-240 (1994).

[37] Zitter, R. N. Saturated optical absorption through band filling in semiconductors. Applied Physics Letters 14, 73-74 (1969).

[38] Hanna, M.C., Zhenghao, L., Nozik, A.J. Hot carrier solar cells. AIP Conference Proceedings 404, 309 (1997). 